\section{P559 INCARCERATION, STRESS AND SEXUAL RISK-TAKING: AN INTERSECTIONAL ANALYSIS OF BLACK MEN WHO HAVE SEX WITH MEN IN THE DEEP SOUTH}

'Denton Callander*, 'Dustin Duncan, 'Su Hyun Park, ${ }^{2}$ Lisa Bowleg, ${ }^{3}$ Lauren BrinkleyRubinstein, ${ }^{4}$ Katherine Theall, ${ }^{5}$ Demarc Hickson. ${ }^{1}$ New York University, School of Medicine, New York, USA; ${ }^{2}$ George Washington University, Washington, USA; ${ }^{3}$ University of North Carolina, Chapel Hill, USA; ${ }^{4}$ Tulane University, New Orleans, USA; ${ }^{5}$ Us Helping US, People Into Living Inc, Washington, USA

10.1136/sextrans-2019-sti.633

Background This study examined if the intersection of stress and experiences of incarceration was associated with sexual risk-taking among Black gay, bisexual and other men who have sex with men ('Black MSM') in the Deep South of the United States.

Methods Data for this analysis were collected during 20132015 as part of a study of sexual health among Black cisgender MSM in the states of Mississippi and Georgia. At baseline, participants responded to a 12-item scale of stressful experiences in the previous year with responses organized into tertiles (low, medium, high stress). Multivariate analyses assessed independent and interactional associations between stress/incarceration and several key sexual health risk practices. Results Among our overall sample of 355 Black MSM, compared to those reporting a relatively low level of stress, a high level of stress was associated with drug use in the context of sex (adjusted odds ratio $[\mathrm{OR}]=2.0,95 \%$ confidence interval $[\mathrm{CI}]: 1.1-3.6, \mathrm{p}=0.03)$. While this association was observed for men without incarceration histories $(\mathrm{aOR}=2.6$, 95\%CI:1.2-5.8, p=0.015), among those who had been incarcerated the relationship was stronger $(\mathrm{aOR}=3.9$, 95\% CI:1.8$8.6, \mathrm{p}<0.001)$ and for these men experiencing even a medium level of stress was associated with sexual drug use $(\mathrm{aOR}=3.4$, 95\%CI:1.6-9.1, $\mathrm{p}<0.001)$. High stress among previously incarcerated MSM was also associated with condomless casual sex $(\mathrm{aOR}=2.8,95 \% \mathrm{CI}: 1.3-6.1, \mathrm{p}<0.001)$ and having $\geq 6$ partners in six months $(\mathrm{aOR}=2.8,95 \% \mathrm{CI}: 2.8-1.1-7.1, \mathrm{p}=0.03)$; similar associations were not observed among men who had not been incarcerated.

Conclusion While stress was associated with some sexual risk taking among Black MSM, its intersection with incarceration was consistently (and more strongly) associated with a greater number and diversity of sexual risk practices. Men who have been incarcerated may struggle to deal with life stressors. Post-release programs for this population should provide adaptive tools for dealing with stress, including specific attention to safer sex and sexual risk.

Disclosure No significant relationships.

\section{P560 \\ HIV PREVENTIVE BEHAVIOUR DURING LAST SEX WITH A NON-STEADY PARTNER - SELF-REPORTED DATA FROM 113,000 MSM FROM 66 COUNTRIES}

${ }^{1}$ Ulrich Marcus*, ${ }^{1}$ Susanne Schink, ${ }^{2}$ Axel Schmidt, ${ }^{3}$ Carlos Caceres, ${ }^{4}$ Kai Jonas, ${ }^{5}$ Maria Veras, ${ }^{6}$ Percy Fernández-Davila, ${ }^{7}$ Henrique Barros, ${ }^{2}$ Peter Weatherburn, ${ }^{2}$ Ford Hickson, ${ }^{2}$ David Reid, ${ }^{6}$ Jordi Casabona I Barbara. ${ }^{1}$ Robert Koch-Institut, Infectious Diseases Epidemiology, Berlin, Germany; ${ }^{2}$ London School of Hygiene and Tropical Medicine, Sigma Research, London, UK; ${ }^{3}$ Universidad Peruana Cayetano Heredia, Center for Interdisciplinary Studies in Sexuality, AIDS and Society, Lima, Peru; ${ }^{4}$ Maastricht University, Department of Work and Social Psychology, Maastricht, Netherlands; ${ }^{5}$ Faculdade de Ciências Médicas da Santa Casa de São Paulo, São Paulo, Brazil; ${ }^{6}$ Centre d'Estudis Epidemiològics sobre les Infeccions de Transmissió Sexual i SIDA de Catalunya, Research Department, Barcelona, Spain; 'University of Porto, Institute of Public Health, Porto, Portugal

\subsection{6/sextrans-2019-sti.634}

Background In Europe and the Americas, men-who-have-sexwith-men (MSM) are heavily affected by HIV. Having traditionally relied mainly on condoms and sero-adaptive behaviours to prevent HIV transmission, more recently biomedical prevention strategies such as pre-exposure prophylaxis (PrEP) among HIV-negative and viral suppression among HIV-diagnosed men have been adopted. We analysed the choice of HIV prevention behaviour (HIV-PB) and determinants of the lack of defined HIV-PB during sex among MSM across 66 countries.

Methods Between October 2017 and May 2018, self-reported data were collected online: EMIS-2017 in 48 European countries (including Lebanon and Israel), and LAMIS in 18 predominantly Latin American countries. Here we focus on men who reported on their last anal sex with a non-steady partner. We used a multilevel multivariate regression model (MMRM) to identify demographic and contextual factors associated with the lack of HIV-PB such as condom use, undetectable viral load, PrEP use or serostatus disclosure.

Results Our analysis included 71,123 MSM from EMIS-2017 and 41,834 MSM from LAMIS. Condom use remained the primary HIV-PB, followed by sero-adaptive behaviours. Using antiretrovirals as HIV-PB accounted for 14.9\% (HIV-diagnosed men: 83.4\%; other MSM: 3.1\%). No HIV-PB as defined above was reported by $20.5 \%$, ranging from 12.1 to $39.7 \%$ across countries. In the MMRM, increasing age, false assumptions about HIV recognisability, struggling financially, living in smaller settlements, having intercourse in a gay sex venue, a group-sex setting, and receiving money for sex were associated with increased odds for lack of HIV-PB. The strongest associations were observed among those who had never been tested for HIV (OR=1.86, 95\%CI: 1.79-1.94) and those who had not bought or received condoms in the last 12 months $(\mathrm{OR}=2.75$; 95\%CI: 2.60-2.91).

Conclusion About 1-in-5 MSM did not use HIV-PB. While antiretrovirals for treatment and prophylaxis gain importance, HIV-testing and condom promotion remain essential to encourage HIV-PB.

Disclosure No significant relationships. 